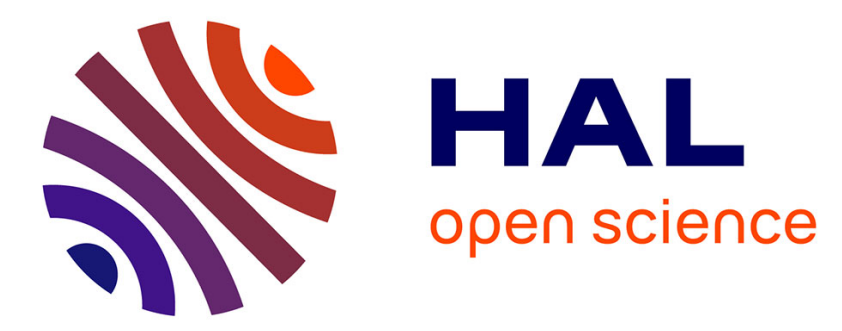

\title{
Nutritional compensation to exercise- vs. diet-induced acute energy deficit in adolescents with obesity
}

David Thivel, Eric Doucet, Valérie Julian, Charlotte Cardenoux, Yves Boirie, Martine Duclos

\section{- To cite this version:}

David Thivel, Eric Doucet, Valérie Julian, Charlotte Cardenoux, Yves Boirie, et al.. Nutritional compensation to exercise- vs. diet-induced acute energy deficit in adolescents with obesity. Physiology \& behavior, 2017, 176, pp.159-164. 10.1016/j.physbeh.2016.10.022 . hal-01594266

\section{HAL Id: hal-01594266 \\ https://hal.science/hal-01594266}

Submitted on 26 Sep 2017

HAL is a multi-disciplinary open access archive for the deposit and dissemination of scientific research documents, whether they are published or not. The documents may come from teaching and research institutions in France or abroad, or from public or private research centers.
L'archive ouverte pluridisciplinaire HAL, est destinée au dépôt et à la diffusion de documents scientifiques de niveau recherche, publiés ou non, émanant des établissements d'enseignement et de recherche français ou étrangers, des laboratoires publics ou privés. 


\title{
Nutritional compensation to exercise- $v$ s. diet-induced acute energy deficit in adolescents with obesity
}

\author{
David Thivel a,b,*, Eric Doucet ${ }^{\mathrm{c}}$, Valérie Julian ${ }^{\mathrm{b}, \mathrm{d}, \mathrm{e}, \mathrm{h}}$, Charlotte Cardenoux ${ }^{\mathrm{f}}$, \\ Yves Boirie ${ }^{\text {be, }, \mathrm{g}, \mathrm{h}}$, Martine Duclos $\mathrm{b,d,e,h}$ \\ a Clermont Auvergne University, EA 3533, Laboratory of the Metabolic Adaptations to Exercise under Physiological and Pathological Conditions (AME2P), Clermont-Ferrand, France \\ b CRNH-Auvergne, Clermont-Ferrand, France \\ c School of Human Kinetics, University of Ottawa, Ottawa, Canada \\ d Department of Sport Medicine and Functional Explorations, Clermont-Ferrand University Hospital, G. Montpied Hospital, Clermont-Ferrand, France \\ e INRA, UMR 1019, Clermont-Ferrand, France \\ ${ }^{\mathrm{f}}$ Romagnat Pediatric Medical Center, Childhood Obesity Department, Romagnat, France \\ ${ }^{g}$ Department of Human Nutrition, Clermont-Ferrand University Hospital, G. Montpied Hospital, Clermont-Ferrand, France \\ ${ }^{\text {h }}$ University Clermont 1, UFR Medicine, Clermont-Ferrand, France
}

\section{H I G H L I G H T S}

- Obese adolescents increase intake in response to energy deficit.

- A 25\% exercise-energy deficit leads to higher overall intake than a similar diet one.

- The extent for the deficit might be responsible for the compensatory intake.

\section{A R T I C L E I N F O}

\section{Article history:}

Received 25 August 2016

Received in revised form 31 October 2016

Accepted 31 October 2016

Available online $\mathrm{xxxx}$

\section{Keywords:}

Energy intake

Exercise

Energy deficit

Pediatric obesity

\begin{abstract}
A B S T R A C T
Background: To compare the energy and macronutrient intake responses to equivalent energy deficits induced by diet (food restriction) and exercise in adolescents with obesity.

Methods: Fourteen 12-15 years old obese adolescents completed three experimental conditions (08:00 am to 07:30 pm) in a randomized crossover design: i) control session (CON); ii) diet-induced 25\% energy depletion (Def-EI), iii) and an exercise-induced 25\% energy depletion (Def-EX). The sessions order was either CON/Def$\mathrm{EI} /$ Def-EX or CON/Def-EX/Def-EI as the deficit corresponded to 25\% of the energy ingested at lunch on the control day (CON) and was imposed either by exercise (Def-EX) or diet (Def-EI). Ad libitum EI and macronutrients preferences were assessed at dinner and appetite sensations assessed using visual analogue scales.

Results: Mean BMI was $36.6 \pm 5.0 \mathrm{~kg} / \mathrm{m}^{2}$ (z-BMI: $2.40 \pm 0.29$ ). The individually calibrated $25 \%$ energy deficit represented $254 \pm 92 \mathrm{kcal}$. Ad libitum EI was significantly higher during both Def-EX ( $971 \pm 225 \mathrm{kcal})$ and Def-EI $(949 \pm 246 \mathrm{kcal})$ compared with CON $(742 \pm 297)(\mathrm{p}<0.05)$. The relative energy ingested derived from fat was significantly higher on both Def-EX (36.6 $\pm 10.9 \%)$ and Def-EI $(36.9 \pm 13.1 \%)$ compared with CON $(21.6 \pm 7.8 \%)(p<0.05)$. The energy derived from carbohydrates was significantly lower on both Def-EX $(48.3 \pm 9.0 \%)$ and Def-EI $(44.4 \pm 17.3 \%)$ compare with CON $(61.1 \pm 10.1 \%)(\mathrm{p}<0.05)$. Appetite sensations were not different between conditions. The induced energy deficit was negatively correlated with the ad libitum EI difference between the exercise and the control session (EI Def-EX - EI CON) $(r=-0,643 p<0.05)$ and positively correlated with the EI difference between the dietary restriction and the control session (EI Def-EI - EI CON) $(r=0,569 p<0.05)$.

Conclusion: Equicaloric exercise- or diet-induced energy deficits could lead to similar EI compensation in obese adolescents but this EI compensation might be influenced by the magnitude of the deficit.
\end{abstract}

(c) 2016 Elsevier Inc. All rights reserved.

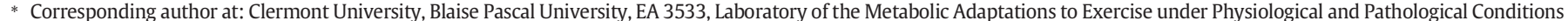
(AME2P), BP 80026, F-63171 Aubière cedex, France.

E-mail address: David.Thivel@univ-bpclermont.fr (D. Thivel).
} 


\section{Introduction}

Pediatric overweight, obesity, and their metabolic complications, are of public health concern and their constant progression highlights the urgent need for new and effective preventive strategies and weight loss programs. Preventing and treating obesity rests on the control of the energy balance, mainly by creating energy deficits through decreased energy intake and/or increased energy expenditure (through increased physical activity participation). While energy intake and expenditure have long been considered independently in the context of body weight control, there is increasing evidence to support that one could indirectly induce compensatory responses to the other $[1,2]$. Mayer and collaborators reported in the mid-fifties in both human [3] and animal models [4] that any increased-energy expenditure was met equally by increased energy intake, so that body weight remained stable, Since then, investigations of the compensatory mechanisms between expenditure and intake have been the subject of many studies $[1,2]$.

In 1998, Hubert et al. compared for the first time the nutritional responses to an equivalent energy deficit induced by dietary restriction or exercise in healthy adults [5]. Interestingly, their results indicated that while an acute energy depletion induced by dietary restriction led to increased hunger feelings and energy intake at the following meal, in contrast the energy deficit induced by a bout of moderate exercise did not significantly alter perceived hunger and did not induce an increase in energy intake at the test lunch [5]. In a more recent study conducted in a similar population, these divergent short term appetite and intake responses to diet- or exercise-induced energy depletion have been attributed to changes in acylated ghrelin and $\mathrm{PYY}_{3-36}$ concentrations that have been found sensitive to the nature of the generated depletion (exercise or diet) [6]. Indeed, while acylated ghrelin has been found higher in the food-restriction day, $\mathrm{PYY}_{3-36}$ was found lower compared with the exercise condition [6]. Interestingly such compensatory responses have been found similar in healthy men and women [7].

Recent studies have questioned the impact of exercise-induced energy deficit on subsequent energy intake in children and adolescents [8-12]. While it has been clearly described that an acute bout of intensive exercise favors a transient anorexigenic effect in obese but not lean adolescents [13], this has been found uncoupled to the energy expended during exercise [14]. There is to our knowledge no study comparing the appetite and energy intake responses to an equicaloric deficit induced either by exercise or dietary restriction in youth.

The aim of the present study was thus to compare the energy intake and appetite sensations responses to equivalent energy deficits induced by diet (food restriction) and exercise in adolescents with obesity. We hypothesized that the adolescents would compensate for the induced energy-deficit by increasing their energy intake on the dietary restriction day only and not to the exercise one.

\section{Materials and methods}

\subsection{Participants}

Fourteen obese (as defined by Cole et al., 2000 [15]) adolescents aged $12-15$ years old (Tanner stage $3-4$ as assessed by the pediatrician after medical examination) took part in this study. Obese adolescents were recruited through pediatric consultations (Clermont-Ferrand University Hospital and Romagnat Children Medical Center, France). To be included in the study, participants had to be free of any medication that could interact with the protocol, could not present any contraindications to physical activity, and had to take part in $<2$ h of physical activity per week (According to the International Physical Activity Questionnaire - IPAQ) and had to be free of any food intolerance and/ or aversion. All adolescents and their legal guardian received information sheets and signed consent forms as requested by the Helsinki declaration and the ethical authorities (CPP Sud Est VI).

\subsection{Overview of the study protocol}

First, a pediatrician performed medical inclusion visit to confirm the eligibility of participants. The adolescents had to fill in a food preference questionnaire during this first visit. They were then required to perform a maximal aerobic test and body composition was also assessed by dualenergy X-ray absorptiometry (DXA) at this time (when included in the study). The adolescents then visited the laboratory on 3 separate occasions (at least 7 days apart): 1) a control session (CON); 2) an exercise-induced energy deficit session (Def-EX); 3 ) a diet-induced energy deficit (Def-EI). The sessions were realized following a crossover design with the sequence of testing being either CON/Def-EI/Def-EX or CON/Def-EX/Def-EI (Fig. 1). Indeed, to calibrate the energy deficit, the CON session had to be realized first. A 25\% energy deficit was imposed either by exercise (Def-EX) or diet (Def-EI) based on the energy ingested at lunch time on the control day (CON) (as previously described [6]). Ad libitum energy intake and appetite sensations were assessed using visual analogue scales at regular intervals through the days for the 3 experimental conditions (the main objective of this study, measuring their energy intake, was not detailed to the adolescents to avoid any influence). The adolescents were asked to avoid any moderate-to-vigorous physical exercise and to consume the same types of meals on the $24 \mathrm{~h}$ before each experimental session (their activity and intake were reported to a member of the investigation team on the first experimental day and this investigator gave advices to make the adolescents follow these instructions on the day prior to the two other sessions).

\subsection{Description of the experimental sessions}

\subsubsection{Control session (CON)}

After ingestion a standardized breakfast (08:30), the adolescents rested for $3 \mathrm{~h} 30$ hours. On the control day, both lunch and dinner (12:00 and 06:30 pm) were offered ad libitum to the adolescents. During the day, the adolescents were asked to remain quiet, not engaging in any moderate to intense physical activities (they were able to read, watch television, do their homework, etc., using the facilities proposed in our laboratory that is adapted to pediatric experiments).

\subsubsection{Exercise-induced energy deficit session (Def-EX)}

At 08:30 am the adolescents received a calibrated breakfast. Between 10:30 am and 11:45 am, the adolescents were asked to cycle on an ergocycle at $65 \%$ of their individual $\mathrm{VO}_{2 \max }$. The duration of the exercise was individually calibrated to generate an energy expenditure that corresponded to $25 \%$ of the energy ingested at lunch time on CON (25\% energy deficit). The intensity was controlled using heart rate records and the workload setting on the cycle ergometer, based on the results from the maximal aerobic capacity testing. At lunch time, the adolescents received a buffet identical to what they consumed for lunch on CON. They were then asked to remain quiet for the rest of the day, until 06:30 pm when they were presented with an ad libitum dinner meal.

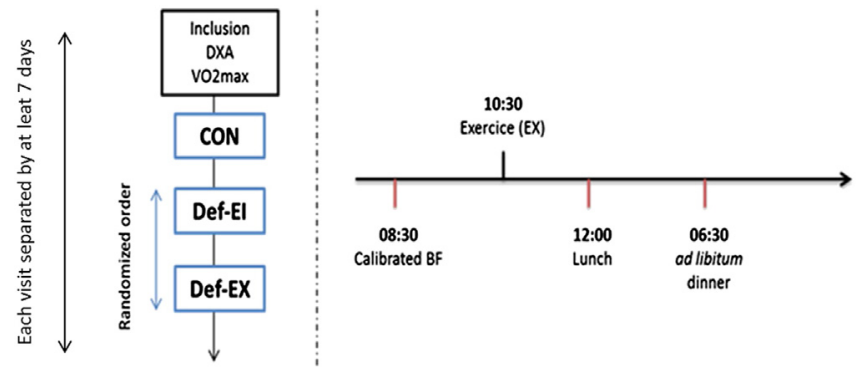

Fig. 1. Study design (CON: condition control; DEF-EX: condition exercise; DEF-EI: dietary restriction condition; BF: breakfast; EX: exercise). 


\subsubsection{Diet-induced energy deficit session (Def-EI)}

At 08:30 am the adolescents received a calibrated breakfast. During the day, the adolescents were asked to remain quiet, not engaging in any moderate to intense physical activities. At 12:00 pm, the lunch served respected the same food items and macronutrient proportion as during CON but was lowered by $25 \%$ (to create a $25 \%$ energy deficit by food restriction). They were then asked to remain quiet for the rest of the day, until 06:30 pm when they were presented with an ad libitum dinner meal.

\subsection{Anthropometric and body composition measurements}

Body weight was measured to the nearest $0.1 \mathrm{~kg}$ using a digital scale, and barefoot standing height was assessed to the nearest $0.1 \mathrm{~cm}$ using a wall-mounted stadiometer. Body mass index (BMI) was calculated as body weight $(\mathrm{kg})$ divided by height squared $\left(\mathrm{m}^{2}\right)$. Fat mass and fatfree mass were assessed using DXA following standardized procedures (QDR4500A scanner, Hologic, Waltham, MA, USA). These measurements were obtained during the preliminary visit by a trained technician.

\subsection{Aerobic capacities}

$\mathrm{VO}_{2 \max }$ was measured during a graded exhaustive cycling test that was performed during a preliminary session at least one week prior to the experimental sessions, by a specialized medical investigator from the Department of Sports Medicine, Functional and Respiratory Rehabilitation (Clermont-Ferrand University Hospital). The initial power was set at $30 \mathrm{~W}$ for $3 \mathrm{~min}$ and followed by $15 \mathrm{~W}$ increments every $1.5 \mathrm{~min}$. Adolescents were strongly encouraged by experimenters throughout the test to perform a maximum effort. Criteria for reaching $\mathrm{VO}_{2 \max }$ were subjective exhaustion with heart rate above 195 beats $\cdot \mathrm{min}^{-1}$ and/or Respiratory Exchange Ratio (RER, $\mathrm{VCO}_{2} / \mathrm{VO}_{2}$ ) above 1.02 and/ or a plateau of $\mathrm{VO}_{2}$ [16]. An electromagnetically-braked cycle ergometer (Ergoline, Bitz, Germany) was used to perform the test. $\mathrm{VO}_{2}$ and $\mathrm{VCO}_{2}$ were measured breath-by-breath through a mask connected to $\mathrm{O}_{2}$ and $\mathrm{CO}_{2}$ analyzers (Oxycon Pro-Delta, Jaeger, Hoechberg, Germany). Calibration of gas analyzers was performed with commercial gases of known concentration. Ventilatory parameters were averaged every 30 s. ECG was monitored for the duration of the test.

\subsection{Energy intake assessment}

On the three experimental days the participants had to consume entirely a standardized breakfast at 08:30 am (same composition and caloric content; composed of one dairy produce; bread and one fruit or fruit juice). This breakfast was composed according to the adolescents' usual breakfast habits and calibrated to reach the nutritional recommendations for their age (total calorie content as well as macronutrient repartition). This method has been previously detailed [11,13].

Lunch energy intake was served ad libitum on CON and the amount of consumed food served to determine the energy deficit induced by the exercise and by the diet (lunch meal). Briefly, the buffet contained at least two choices of each dish (although the buffet were individually composed, green salads, tomato salads, mushed potatoes, pasta, beef, chicken, different kinds of cheeses, yogurts, compote, different kinds of fruits were the main served food items). On Def-EI, the lunch served respected the same food items and macronutrient proportion as during CON but caloric content was reduced by $25 \%$ (to create a $25 \%$ energy deficit by food restriction). Ad libitum buffet meals were served on the three occasions (CON/Def-EX/Def-EI) for dinner. The composition of the buffet was conformed to the adolescents' tastes as determined by a food preference questionnaire completed prior to the experimental sessions (i.e. during the preliminary visit). Briefly, the adolescents had to rate their food preferences and habits among about 100 different food items, top rated items were avoided to limit overconsumption, as generally seen in ad libitum feeding experiments with adolescents. Similarly, usually consumed items only were proposed to avoid any occasional eating by proposing "liked" items but that are rarely consumed by the adolescents. The method used to assess ad libitum energy intake in adolescents with obesity has been previously published [17]. None of the adolescents were excluded on the basis of this questionnaire. During ad libitum meals (lunch CON and the three dinners), participants were told to eat until satisfied; additional food was provided if desired. Food consumption was weighed and recorded by investigators (Bilnut 4.0 SCDA Nutrisoft software, France) to calculate total energy intake during lunch. The proportion of the total energy intake derived from fat, carbohydrate and protein was calculated using the same nutritional software used to assess energy intake (Bilnut 4.0 SCDA Nutrisoft software, France). Ad libitum lunch meal methodology and spontaneous energy intake assessment were previously detailed [11].

\subsection{Subjective appetite sensations}

At regular intervals throughout the day from 08:30 am, participants were asked to rate their hunger, fullness and prospective food consumption using visual analogue scales (VAS of $100 \mathrm{~mm}$ ) whose reliability has been previously reported [18]. Participants filled in VAS before and after the breakfast, before and after lunch, and 30,60 and $120 \mathrm{~min}$ after lunch, before and after dinner. This method has previously been used among obese adolescents to evaluate appetite sensations [12,19].

\subsection{Statistical analysis}

Statistical analyses were performed using Statview 5.0 (SAS Institute, NC, USA). Results are expressed as mean (standard deviation). The distribution of the data was tested using the Smirnov-Kolmogorov test prior to analysis and data did not require any transformations prior to analyses. Repeated measures ANOVA were performed to compare energy and macronutrient intakes and whole-day appetite sensations AUC (Area Under the Curve calculated using the trapezoidal method) between experimental sessions (CON, Def-EX, Def-EI). Hunger, fullness and prospective food consumption just before dinner time (meal time) were also compared between sessions using repeated measures ANOVA. Finally, correlations were performed between the participants' induced absolute energy-deficit (in kcal) and the ad libitum Energy Intake difference between: i) the exercise session and the control session (EI Def-EX - EI CON) and between ii) the dietary restriction session and control session (EI Def-EI - EI CON). None of the above mentioned analysis revealed a gender effect (secondary analyses were performed using gender as a supplementary factor). Moreover, all the performed analyses were adjusted for the participants' body weight and gender. The level of significance was set at $\mathrm{p}<0.05$.

\section{Results}

As presented in Table 1, the 14 adolescents ( 7 girls and 7 agematched boys) had a mean age of $14.2 \pm 1.0$ years old and a BMI of

Table 1

Characteristics of the population.

\begin{tabular}{ll}
\hline & Mean \pm SD \\
\hline Age (years old) & $14,2 \pm 1,0$ \\
Weight $(\mathrm{kg})$ & $103,7 \pm 13,5$ \\
z-BMI & $2.40 \pm 0.29$ \\
BMI $\left(\mathrm{kg} / \mathrm{m}^{2}\right)$ & $36,6 \pm 5,0$ \\
FM $(\%)$ & $41,2 \pm 5,2$ \\
FFM $(\mathrm{kg})$ & $58,4 \pm 8,1$ \\
Central FM (\%) & $45,3 \pm 6,0$ \\
25\% energy deficit (kcal) & $254 \pm 92$ \\
\hline
\end{tabular}

SD: standard deviation; BMI: body mass index; FM: fat mass; FFM: fat free mass. 
$36.6 \pm 5.0 \mathrm{~kg} / \mathrm{m}^{2}$ (z-BMI: $2.40 \pm 0.29$ and BMI percentile: $98.4 \pm 0.9$ ). The individually calibrated 25\% energy deficit represented $254 \pm$ $92 \mathrm{kcal}$. The exercise ranged between 21 and $50 \mathrm{~min}$ between participants.

As illustrated by the Fig. 2B, the adolescents' absolute energy ingested during the ad libitum test meal was significantly higher during both Def-EX ( $971 \pm 225 \mathrm{kcal})$ and Def-EI (949 $\pm 246 \mathrm{kcal})$ compared with CON (742 \pm 297$)$ ( $\mathrm{p}<0.05)$, with no difference between Def-EI and Def-EX. Daily energy intake (lunch + diner) was significantly higher on Def-EX compared with both Con $(p<0.05)$ and Def-EI $(\mathrm{p}<0.05)$, with no difference between Def-EI and CON (Fig. 2C).

While the absolute $\mathrm{CHO}$ and Protein consumption (in grams) at the dinner time test meal was not significantly different between condition, absolute fat intake was significantly higher on Def-EI $(41,3 \pm 24,7 \mathrm{~g})$ and Def-EX $(40,4 \pm 18,4 \mathrm{~g})$ compared with CON $(18,7 \pm 10,2 \mathrm{~g})$ $(\mathrm{p}<0.01)$. The percentage of energy ingested derived from proteins during the test meal was not significantly different between conditions. The relative energy ingested derived from fat was significantly higher on both Def-EX (36.6 $\pm 10.9 \%)$ and Def-EI (36.9 $\pm 13.1 \%)$ compared

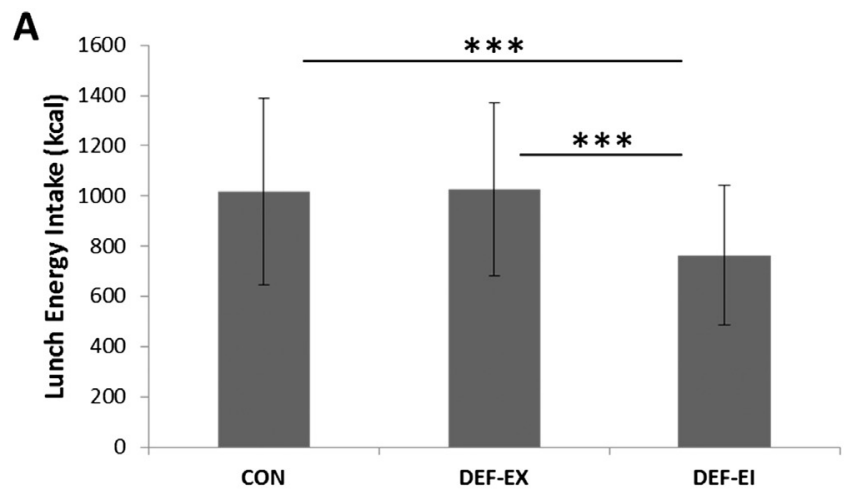

B
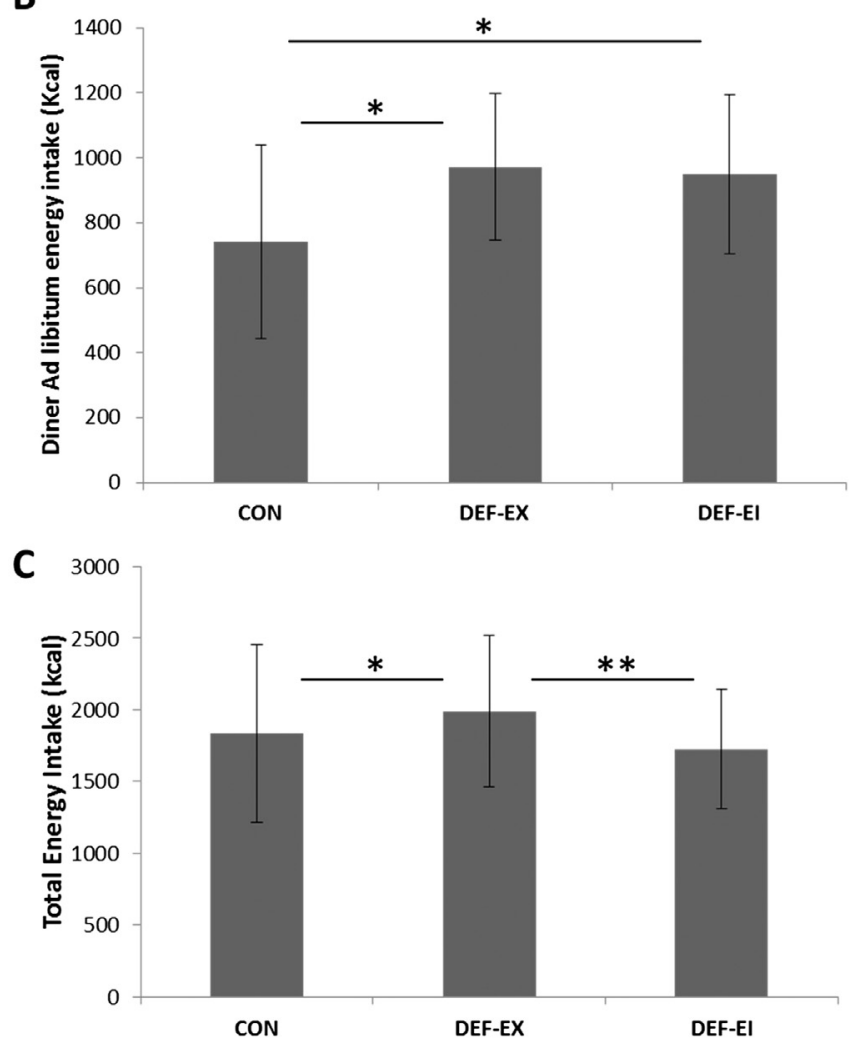

Fig. 2. Energy intake at lunch (A), dinner (ad libitum test meal - B) and through the day (lunch + dinner) (C) (CON: condition control; DEF-EX: condition exercise; DEF-EI: dietary restriction condition ; ${ }^{*} \mathrm{p}<0.05 ;{ }^{* *} \mathrm{p}<0.01$; ${ }^{* * *} \mathrm{p}<0.001$ ). with CON $(21.6 \pm 7.8 \%)(\mathrm{p}<0.05)$. The energy derived from carbohydrates was significantly lower on both Def-EX $(48.3 \pm 9.0 \%)$ and DefEI $(44.4 \pm 17.3 \%)$ compare with CON $(61.1 \pm 10.1 \%)(\mathrm{p}<0.05)$. Results relative to the energy consumed during the ad libitum test meal are presented in Table 2 .

None of the appetite sensations under study (hunger, fullness and prospective food consumption) were significantly different between conditions. When analyzed separately, hunger showed a tendency to be higher just before the ad libitum test meal on both Def-EX (107 \pm $51 \mathrm{~mm})$ and Def-EI $(112 \pm 39 \mathrm{~mm})$ compared with CON $(73 \pm$ $51 \mathrm{~mm})(\mathrm{p}=0.07)$ and fullness was significantly higher on CON (74 $\pm 48 \mathrm{~mm}$ ) compared with the two other sessions (Def-EX: $28 \pm$ $44 \mathrm{~mm}$ and Def-EI: $23 \pm 30)(\mathrm{p}>0.05)$. There was no difference for fasting appetite sensations between conditions.

There are significant correlations between the participants' induced energy-deficit (25\% energy deficit as previously detailed) and the ad libitum Energy Intake difference between the exercise and the control session (EI Def-EX - EI CON) (negative correlation; $r=-0,643$ $\mathrm{p}<0.05$ ) and between the dietary restriction and the control session (EI Def-EI - EI CON) (positive correlation; $r=0,569 p<0.05$ ) (adjusted for body weight).

\section{Discussion}

The objective of this study was to determine whether there are measurable nutritional responses to a tightly controlled energy depletion of $25 \%$ induced by food restriction or exercise in adolescents with obesity. This is to our knowledge the first study to compare energy and macronutrient intakes and appetite sensations responses to a similar energy deficit induced whether by exercise or diet in this population. According to our results, both acute exercise and dietary restriction favored increased ad libitum energy intake at the following test meal compared with a control condition. However, one of the main findings in this study is the different associations observed between the energy restriction induced by exercise or diet and the following compensation, with a negative correlation observed between the degree of induced deficit and the energy intake at the test meal on the exercise day and on the opposite, when the deficit is created using diet, its extent is positively related to the amount of consumed calories at the ad libitum meal.

Although these are the first results in adolescents, they are contradictive to what has been previously found in adults. Adults have effectively been shown to differently respond to a similar energy deficit depending on its origin (exercise or diet) [5-7,20]. According to Hubert and collaborators, who were the first to conduct such a study in healthy adults, an acute energy deficit induced by dietary a low caloric breakfast led to increased hunger feeling and food consumption while the same energy deficit induced by a bout of moderate exercise did not significantly alter perceived hunger and energy intake at the test lunch [5]. Such nutritional responses have been associated to opposite effects of exercise and dietary-induced energy depletion on some of the main hormonal factors controlling appetite and food intake. It has effectively been shown that for a similar energy depletion, the concentrations of

Table 2

Absolute (in grams) an relative (percentage of total ingested energy) consumption of each macronutrients on the ad libitum diner test meal.

\begin{tabular}{llll}
\hline & CON & Def-EX & Def-EI \\
\hline Protein (gr) & $34,4 \pm 23,6$ & $36,6 \pm 11,9$ & $35,2 \pm 13,3$ \\
Lipids (gr) & $18,7 \pm 10,2$ & $40,4 \pm 18,4^{* *}$ & $41,3 \pm 24,7^{* *}$ \\
CHO (gr) & $108,9 \pm 34,8$ & $115,1 \pm 26,2$ & $100,4 \pm 33,3$ \\
Protein (\%) & $17,3 \pm 4,5$ & $14,9 \pm 3,2$ & $14,7 \pm 4,4$ \\
Lipids (\%) & $21,6 \pm 7,8$ & $36,6 \pm 10,9^{*}$ & $36,9 \pm 13,1^{*}$ \\
CHO (\%) & $61,1 \pm 10,1$ & $48,3 \pm 9,0^{*}$ & $44,4 \pm 17,3^{*}$ \\
\hline
\end{tabular}

SD: standard deviations; CON: condition control; Def-EX: condition exercise; Def-EI: dietary restriction condition; EI: energy intake; $\mathrm{CHO}$ : carbohydrate.

* $\mathrm{p}<0.05$ versus CON.

** $\mathrm{p}<0.01$ versus CON 
acylated ghrelin (as an orexigenic actor) were higher and PYY ${ }_{3-36}$ lower (as an anorexigenic actor) on a food-restriction day compared with an exercise day in healthy adult men and women [6,7]. Although the present results are the first in adolescents, their discrepancy with the available literature in adults might also be due to a weight status effect. Indeed, the previously published studies have been conducted among lean adults and there is no such result so far among overweight/obese patients.

Although we observe higher relative lipids and lower carbohydrates consumptions on both the exercise and dietary restriction conditions, only absolute (in grams) lipids ingestion is significantly increased on both deficit days. This remains difficult to discuss since there is no comparable study in the actual literature in youth. Interestingly, while King and collaborators, observed energy compensation after the dietinduced energy depletion only, their results also underline significantly lower $\mathrm{CHO}$ intakes and higher lipid intakes on both exercise and food restriction days in healthy adults compared with the control session [6]. The nature of the induced-energy deficit (exercise versus diet) might then differentially affect macronutrient intake but further studies are needed.

An interesting observation is that appetite sensations (AUC for hunger, fullness and PFC) were not significantly different, but energy intake was nonetheless increased at the test meal for the two energy deficit conditions. This is in line with the already described uncoupled effect of exercise on appetite sensations in youth [21]. Although daily appetite sensations were not different between conditions, hunger tended to be increased and fullness significantly decreased during Def-EI and Def-EX when values measured immediately before the test meal were analyzed, which might have contributed to the higher food ingestion observed.

Despite a significantly higher food consumption at the test meal on both the exercise and diet day, daily energy intake was only significantly higher on Def-EX with no difference between Def-EI and CON and our results suggest a relationship between the degree of induced absolute energy deficit and the following nutritional responses, depending on the nature of the created-deficit (exercise or food restriction). Indeed, it appears that the higher is the deficit and higher is the energy intake at the test meal on the food restriction day. On the opposite, when the deficit is created using exercise, its extent is inversely related to the amount of consumed calories at the ad libitum meal. These results tend to reinforce the hypothesis that exercise is a better way to induce energy deficit compared with dietary restriction by preventing nutritional compensatory responses. However, this would depend on the degree of the energy deficit. The $25 \%$ energy deficit used in the present work might not have been high enough to avoid increased ad libitum energy intake on the exercise day (ranging from 110 to $351 \mathrm{kcal}$ ). It must be reminded here that the energy deficit induced corresponds to $25 \%$ of the energy ingested at lunch time on the CON day and not of daily energy intake. Although post-exercise energy intake has been found uncoupled with the amount of energy expended during the exercise in lean and obese youth [14], one could suggest that it has to create a high enough deficit to affect the neurophysiological signals and pathways involved in the control of energy intake.

While the studies realized so far in this area questioned the acute (over 1 day) effect of exercise- and diet-induced energy deficit on subsequent energy intake [5-7], in their recent randomized controlled trial, Cameron et al. explored such compensatory responses over a 3-day period among healthy young males [20]. According to their results, food restriction represents a greater challenge to appetite compared with an equivalent deficit induced by exercise, with greater appetite and ad libitum energy intake [20]. It appears today necessary to conduct longer study since it is well established that the energy deficit induced by exercise is not compensated for by adaptations of energy intake and appetite within the first days or weeks $[2,22]$.

Some limitations have to be considered when interpreting the present results. First, as previously mentioned the $25 \%$ energy deficit induced at lunch time might not have been sufficiently high and/or prolonged enough in some participants to affect the physiological pathways controlling energy intake and appetite. Further studies employing graded energy deficit within participants should be conducted to answer such a question. To ensure the ability of all the participants to complete the exercise session, a moderate-intensity exercise was implemented while only intense exercise ( $>70 \%$ of the maximal capacities) has been shown to induce a transient anorexigenic effect in this population $[8,9,12]$. Caution must also be used when interpreting and extrapolating laboratory-based studies into free-living environment, even if the exercise used in the present work is similar to what is usually prescribed to obese adolescents as part of their physical activity program. Similarly, the laboratory setting might have impacted the adolescents' energy intake as compared with free-living conditions. The method used to assess energy intake has been however previously shown accurate and reproducible among adolescents with obesity [17]. Finally, the dietary status of the adolescents (restrain/unrestrained for instance) was not assessed in the present study, which might influence such results, and further studies involving lean adolescents should be conducted to provide some evidence regarding a potential weightstatus effect.

In conclusion, while these preliminary results might indicate that equicaloric exercise- or diet-induced energy deficits could lead to similar energy intake compensation in adolescents with obesity; this might be dependent on the degree of the induced-energy deficit. Since this is the first work in this area in youth, further studies using stronger designs comparing different levels of energy deficit should be conducted.

\section{Conflict of interest}

The authors have no conflicts of interest to disclose. The authors have no financial relationships relevant to this article to disclose.

\section{Acknowledgements}

The authors want to thank the adolescents who took part in the study as well as Miss Nais Petiot and Miss Audrey Marion for their help.

\section{References}

[1] J.E. Blundell, et al., Appetite control and energy balance: impact of exercise, Obes. Rev. 16 (Suppl. 1) (2015) 67-76.

[2] C. Drenowatz, Reciprocal compensation to changes in dietary intake and energy expenditure within the concept of energy balance, Adv. Nutr. 6 (5) (2015) 592-599.

[3] J. Mayer, P. Roy, K.P. Mitra, Relation between caloric intake, body weight, and physical work: studies in an industrial male population in West Bengal, Am. J. Clin. Nutr. 4 (2) (1956) 169-175.

[4] M.J. Fregly, N.B. Marshall, J. Mayer, Effect of changes in ambient temperature on spontaneous activity, food intake and body weight of goldthioglucose-obese and nonobese mice, Am. J. Phys. 188 (3) (1957) 435-438.

[5] P. Hubert, N.A. King, J.E. Blundell, Uncoupling the effects of energy expenditure and energy intake: appetite response to short-term energy deficit induced by meal omission and physical activity, Appetite 31 (1) (1998) 9-19.

[6] J.A. King, et al., Differential acylated ghrelin, peptide YY3-36, appetite, and food intake responses to equivalent energy deficits created by exercise and food restriction, J. Clin. Endocrinol. Metab. 96 (4) (2011) 1114-1121.

[7] N. Alajmi, et al., Appetite and energy intake responses to acute energy deficits in females versus males, Med. Sci. Sports Exerc. 48 (3) (2016) 412-420.

[8] S. Tamam, et al., Overweight and obese boys reduce food intake in response to a glucose drink but fail to increase intake in response to exercise of short duration, Appl. Physiol. Nutr. Metab. 37 (3) (2012) 520-529.

[9] N.C. Bozinovski, et al., The effect of duration of exercise at the ventilation threshold on subjective appetite and short-term food intake in 9 to 14 year old boys and girls, Int. J. Behav. Nutr. Phys. Act. 6 (2009) 66.

[10] W.L. Prado, et al., Effect of aerobic exercise on hunger feelings and satiety regulating hormones in obese teenage girls, Pediatr. Exerc. Sci. 26 (4) (2015) 463-469.

[11] D. Thivel, et al., Intensive exercise: a remedy for childhood obesity? Physiol. Behav. 102 (2) (2011) 132-136.

[12] D. Thivel, et al., The 24-h energy intake of obese adolescents is spontaneously reduced after intensive exercise: a randomized controlled trial in calorimetric chambers, PLoS One 7 (1) (2012), e29840.

[13] D. Thivel, et al., Obese but not lean adolescents spontaneously decrease energy intake after intensive exercise, Physiol. Behav. 123 (2014) 41-46. 
[14] D. Thivel, et al., Daily energy balance in children and adolescents. Does energy expenditure predict subsequent energy intake? Appetite 60 (1) (2013) 58-64.

[15] T.J. Cole, et al., Establishing a standard definition for child overweight and obesity worldwide: international survey, BMJ 320 (7244) (2000) 1240-1243.

[16] T.W. Rowland, Developmental exercise physiology, Human Kinetics, 1996 (Champaign, IL).

[17] D. Thivel, et al., Reproducibility of an in-laboratory test meal to assess ad libitum energy intake in adolescents with obesity, Appetite 105 (2016) 129-133.

[18] A. Flint, et al., Reproducibility, power and validity of visual analogue scales in assessment of appetite sensations in single test meal studies, Int. J. Obes. Relat. Metab. Disord. 24 (1) (2000) 38-48.
[19] D. Thivel, et al., Gender effect on exercise-induced energy intake modification among obese adolescents, Appetite 56 (3) (2011) 658-661.

[20] J.D. Cameron, et al., Energy depletion by diet or aerobic exercise alone: impact of energy deficit modality on appetite parameters, Am. J. Clin. Nutr. 103 (4) (2016) 1008-1016.

[21] D. Thivel, J.P. Chaput, Are post-exercise appetite sensations and energy intake coupled in children and adolescents? Sports Med. 44 (6) (2014) 735-741.

[22] J.E. Donnelly, et al., Does increased exercise or physical activity alter ad-libitum daily energy intake or macronutrient composition in healthy adults? A systematic review, PLoS One 9 (1) (2014), e83498. 